\title{
Single Peak Solitons for the Boussinesq-Like $B(2,2)$ Equation
}

\author{
Lina Zhang, ${ }^{1}$ Shumin $\mathrm{Li}^{2}$ and Aiyong $\mathrm{Chen}^{3}$ \\ ${ }^{1}$ School of Science, Huzhou University, Huzhou, Zhejiang 313000, China \\ ${ }^{2}$ Center for Nonlinear Science Studies, Kunming University of Science and Technology, Kunming, \\ Yunnan 650093, China \\ ${ }^{3}$ School of Mathematics and Computing Science, Guilin University of Electronic Technology, \\ Guilin, Guangxi 541004, China \\ Correspondence should be addressed to Lina Zhang; zsdzln@126.com
}

Received 7 April 2013; Revised 30 August 2013; Accepted 3 September 2013

Academic Editor: Athanasios N. Yannacopoulos

Copyright (C) 2013 Lina Zhang et al. This is an open access article distributed under the Creative Commons Attribution License, which permits unrestricted use, distribution, and reproduction in any medium, provided the original work is properly cited.

The nonlinear dispersive Boussinesq-like $B(2,2)$ equation $u_{t t}+\left(u^{2}\right)_{x x}-\left(u^{2}\right)_{x x x x}=0$, which exhibits single peak solitons, is investigated. Peakons, cuspons and smooth soliton solutions are obtained by setting the $B(2,2)$ equation under inhomogeneous boundary condition. Asymptotic behavior and numerical simulations are provided for these three types of single peak soliton solutions of the $B(2,2)$ equation.

\section{Introduction}

The $B(2,2)$ interest inspired by the well-known CamassaHolm $(\mathrm{CH})$ equation and its singular peakon solutions [1] prompted search for other integrable equations with nonsmooth solitons. An integrable $\mathrm{CH}$-type equation with cubic nonlinearity

$$
m_{t}+\left[m\left(u^{2}-u_{x}^{2}\right)\right]_{x}=0, \quad m=u-u_{x x}
$$

was derived independently by Fokas [2], by Fuchssteiner [3], by Olver and Rosenau [4], and by Qiao [5]. It is shown in [57] that (1) admits Lax pair and bi-Hamiltonian structures and possesses the M/W-shape soliton solution and a new type of cusped soliton solution. Another peakon equation with cubic nonlinearity has been recently discovered by Novikov [8]. In the work by Hone and Wang [9], it is shown that Novikov's equation admits peakon solutions like the $\mathrm{CH}$ equation. Also, it has a Lax pair in matrix form and a bi-Hamiltonian structure.

The Boussinesq-like $B(m, n)$ equation with nonlinear dispersion is given by

$$
u_{t t}+a\left(u^{n}\right)_{x x}+b\left(u^{m}\right)_{x x x x}=0,
$$

where $a, b \in R$ and $m, n \in Z^{+}$. This equation is the generalized form of the Boussinesq equation, where, in particular, the case $(m, n)=(1,2)$ leads to the Boussinesq equation. Equation (2), for $a b>0$, is the major equation for compactons (solitons with compact support). Abundant compactons [1013] are developed by the Adominan decomposition method. For $a b<0$, exact solutions with solitary patterns of Boussinesq-like $B(m, n)$ equations are obtained in the works by Shang [14] and Zhang et al. [15] by extending sinh-cosh method and by using the integral approach, respectively.

A natural question is that whether the Boussinesq-like $B(m, n)$ equation (2) has nonsmooth solitons such as peakons or cuspons. The present paper focuses on the following Boussinesq-like $B(2,2)$ equation:

$$
u_{t t}+\left(u^{2}\right)_{x x}-\left(u^{2}\right)_{x x x x}=0
$$

We give all possible single peak soliton solutions of (3) through setting the traveling wave solution under the inhomogeneous boundary condition $u \rightarrow A$ ( $A$ is a nonzero constant) as $x \rightarrow \pm \infty$. New cusped soliton solutions, and smooth soliton solutions are obtained. Asymptotic analysis and numerical simulations are provided for peaked solitons, cusped solitons and smooth solitons of the $B(2,2)$ equation. The method used here is based on the phase portrait analysis technique which is similar to that in [16-18]. 


\section{Asymptotic Behavior of Solutions}

In this section, we first introduce some notations. Let $C^{k}(\Omega)$ denote the set of all $k$ times continuously differential functions on the open set $\Omega . L_{\text {loc }}^{p}(R)$ refers to the set of all functions whose restriction on any compact subset is $L^{p}$ integrable. $H_{\text {loc }}^{1}(R)$ stands for $H_{\text {loc }}^{1}(R)=\left\{u \in L_{\text {loc }}^{2}(R) \mid u^{\prime} \in\right.$ $\left.L_{\text {loc }}^{2}(R)\right\}$.

Let us consider the traveling wave solution of the $B(2,2)$ equation (3) through the setting $u(x, t)=U(x-c t)$, where $c$ is the wave speed. Let $\xi=x-c t$; then $u(x, t)=U(\xi)$. Substituting it into (3) yields

$$
c^{2} U^{\prime \prime}+\left(U^{2}\right)^{\prime \prime}-\left(U^{2}\right)^{\prime \prime \prime \prime}=0
$$

where " $"$ " is the derivative with respect to $\xi$. Integrating (4) once and neglecting the integration constant, we have

$$
c^{2} U^{\prime}+\left(U^{2}\right)^{\prime}-\left(U^{2}\right)^{\prime \prime \prime}=0 .
$$

Integrating (5) once again, we obtain

$$
c^{2} U+U^{2}-2 U^{\prime 2}-2 U U^{\prime \prime}=g_{1},
$$

where $g_{1}$ is an integration constant. Furthermore, we get

$$
U^{\prime 2}=\frac{U^{2}}{4}+\frac{c^{2} U}{3}-\frac{g_{1}}{2}+\frac{g_{2}}{U^{2}},
$$

where $g_{2}$ is also an integration constant.

To seek exact solutions with solitary patterns for (7), we impose the boundary condition

$$
\lim _{\xi \rightarrow \pm \infty} U(\xi)=A,
$$

where $A$ is a nonzero constant. Equation (7) can be cast into the following ordinary differential equation:

$$
U^{\prime 2}=\frac{(U-A)^{2}\left[3 U^{2}+\left(6 A+4 c^{2}\right) U+3 A^{2}+2 A c^{2}\right]}{12 U^{2}} .
$$

The fact that both sides of (9) are nonnegative implies that $3 U^{2}+\left(6 A+4 c^{2}\right) U+3 A^{2}+2 A c^{2} \geq 0$. If $3 A+2 c^{2} \geq 0$, then (9) reduces to

$$
U^{\prime 2}=\frac{(U-A)^{2}\left(U-B_{1}\right)\left(U-B_{2}\right)}{4 U^{2}},
$$

where

$$
\begin{aligned}
& B_{1}=-\frac{3 A+2 c^{2}}{3}+\frac{\sqrt{2 c^{2}\left(3 A+2 c^{2}\right)}}{3}, \\
& B_{2}=-\frac{3 A+2 c^{2}}{3}-\frac{\sqrt{2 c^{2}\left(3 A+2 c^{2}\right)}}{3} .
\end{aligned}
$$

Obviously, $B_{1} \geq B_{2}$.

Definition 1. A function $u(x, t)=U(x-c t)$ is said to be a single peak soliton solution for the $B(2,2)$ equation (3) if $U(\xi)$ satisfies the following conditions.
(A1) $U(\xi)$ is continuous on $R$ and has a unique peak point $\xi_{0}$, where $u(\xi)$ attains its global maximum or minimum value.

(A2) $U(\xi) \in C^{4}\left(R-\left\{\xi_{0}\right\}\right)$ satisfies (8) on $R-\left\{\xi_{0}\right\}$.

(A3) $\lim _{\xi \rightarrow \pm \infty} U(\xi)=A$.

Definition 2. A wave function $U$ is called peakon if $U$ is smooth locally on either side of $\xi_{0}$ and $\lim _{\xi \uparrow \xi_{0}} U^{\prime}(\xi)=$ $-\lim _{\xi \downarrow \xi_{0}} U^{\prime}(\xi)=a, a \neq 0, a \neq \pm \infty$.

Definition 3. A wave function $U$ is called cuspon if $U$ is smooth locally on either side of $\xi_{0}$ and $\lim _{\xi \uparrow \xi_{0}} U^{\prime}(\xi)=$ $-\lim _{\xi \downarrow \xi_{0}} U^{\prime}(\xi)= \pm \infty$.

Without any loss of generality, we choose the peak point $\xi_{0}$ as vanishing, $\xi_{0}=0$.

Theorem 4. Suppose that $U(\xi)$ is a single peak soliton solution for the $B(2,2)$ equation (3) at the peak point $\xi_{0}=0$. Then one has the following.

(i) If $A<-2 c^{2} / 3$, then $U(0)=0$.

(ii) If $A \geq-2 c^{2} / 3$, then $U(0)=0$ or $U(0)=B_{1}$ or $U(0)=$ $B_{2}$.

Proof. If $U(0) \neq 0$, then $U(\xi) \neq 0$ for any $\xi \in R$ since $U(\xi) \in$ $C^{4}(R-\{0\})$. Differentiating both sides of (9) yields $U(\xi) \in$ $C^{\infty}(R)$

(i) For $A<-2 c^{2} / 3$, if $U(0) \neq 0$, then $U \in C^{\infty}(R)$. By the definition of single peak soliton, we have $U^{\prime}(0)=$ 0 . However, by (9) we must have $U(0)=A$, which contradicts the fact that 0 is the unique peak point.

(ii) For $A \geq-2 c^{2} / 3$, if $U(0) \neq 0$, by (9) we know that $U^{\prime}(0)$ exists. According to the definition of peak point, we have $U^{\prime}(0)=0$. Thus we obtain $U(0)=B_{1}$ or $U(0)=$ $B_{2}$ from (10) since $U(0)=A$ contradicts the fact that 0 is the unique peak point.

Theorem 5. Suppose that $U(\xi)$ is a single peak soliton solution for the $B(2,2)$ equation (3) at the peak point $\xi_{0}=0$. Then one has the following solutions classification and asymptotic behavior.

(i) If $U(0) \neq 0$, then $U(\xi)$ is a smooth soliton solution.

(ii) If $U(0)=0$ and $A=-2 c^{2} / 3$, then $U(\xi)$ gives the peaked soliton solution $\left(-2 c^{2} / 3\right)\left(1-e^{-|x-c t| / 2}\right)$.

(iii) If $U(0)=0$ and $A \neq-2 c^{2} / 3$, then $U(\xi)$ is a cusped soliton solution and

$$
\begin{gathered}
U(\xi)=\mu|\xi|^{1 / 2}+O(|\xi|), \quad \xi \longrightarrow 0, \\
U^{\prime}(\xi)=\frac{\mu}{2}|\xi|^{-1 / 2} \operatorname{sgn}(\xi)+O(1), \quad \xi \longrightarrow 0,
\end{gathered}
$$

where $\mu= \pm(1 / 3) \sqrt{3|A| \sqrt{9 A^{2}+6 A c^{2}}}$. Thus $U(\xi) \notin H_{\text {loc }}^{1}(R)$. 
Proof. (i) If $U(0) \neq 0$, then $U(\xi) \in C^{\infty}(R)$ for any $\xi \in R$, and so $U(\xi)$ is a smooth soliton solution.

(ii) If $U(0)=0$ and $A=-2 c^{2} / 3$, then (9) becomes

$$
U^{\prime 2}=\frac{1}{4}\left(U+\frac{2 c^{2}}{3}\right)^{2}
$$

Solving (13), we obtain the peaked soliton solution

$$
U(\xi)=-\frac{2 c^{2}}{3}\left(1-e^{-|x-c t| / 2}\right)
$$

(iii) If $U(0)=0$ and $A \neq-2 c^{2} / 3$, then by the definition of single peak soliton solution we have $A \neq 0$; thus, $3 U^{2}+(6 A+$ $\left.4 c^{2}\right) U+3 A^{2}+2 A c^{2}$ does not contain the factor $U$. From (9), we obtain

$$
\begin{aligned}
U^{\prime}= & -\operatorname{sgn}(A) \frac{U-A}{U} \\
& \times \sqrt{\frac{3 U^{2}+\left(6 A+4 c^{2}\right) U+3 A^{2}+2 A c^{2}}{12}} \operatorname{sgn}(\xi) .
\end{aligned}
$$

Let $\quad h(U)=2 \sqrt{3} \operatorname{sgn}(A) /(A \quad-$ $U) \sqrt{3 U^{2}+\left(6 A+4 c^{2}\right) U+3 A^{2}+2 A c^{2}}$; then $h(0)=$ $2 \sqrt{3} /|A| \sqrt{3 A^{2}+2 A c^{2}}$ and

$$
\int U h(U) d U=\int \operatorname{sgn}(\xi) d \xi .
$$

Inserting $h(U)=h(0)+O(U)$ into (16) and using the initial condition $U(0)=0$, we obtain

$$
\frac{h(0)}{2} U^{2}(1+O(U))=|\xi| \text {. }
$$

Thus

$$
\begin{aligned}
U & = \pm \sqrt{\frac{2}{h(0)}}|\xi|^{1 / 2}(1+O(U))^{-1 / 2} \\
& = \pm \sqrt{\frac{2}{h(0)}}|\xi|^{1 / 2}(1+O(U)),
\end{aligned}
$$

which implies that $U=O\left(|\xi|^{1 / 2}\right)$. Therefore, we have

$$
\begin{gathered}
U(\xi)=\mu|\xi|^{1 / 2}+O(|\xi|), \quad \xi \longrightarrow 0, \\
U^{\prime}(\xi)=\frac{\mu}{2}|\xi|^{-1 / 2} \operatorname{sgn}(\xi)+O(1), \quad \xi \longrightarrow 0,
\end{gathered}
$$

where $\mu= \pm(1 / 3) \sqrt{3|A| \sqrt{9 A^{2}+6 A c^{2}}}$. Thus $U(\xi) \notin H_{\mathrm{loc}}^{1}(R)$.

\section{Peakons, Cuspons, and Smooth Soliton Solutions}

Theorem 5 gives a classification for all single peak soliton solutions for the $B(2,2)$ equation (3). In this section, we will present all possible single peak soliton solutions. We should discuss three cases: $A>-2 c^{2} / 3, A=-2 c^{2} / 3$, and $A<-2 c^{2} / 3$.

Case I $\left(A>-2 c^{2} / 3\right)$. By virtue of Theorems 4 and 5 , any single peak soliton solution for the $B(2,2)$ equation (3) must satisfy the following initial and boundary values problem:

$$
\begin{gathered}
U^{\prime 2}=\frac{(U-A)^{2}\left(U-B_{1}\right)\left(U-B_{2}\right)}{4 U^{2}}, \\
U(0) \in\left\{0, B_{1}, B_{2}\right\}, \\
\lim _{\xi \rightarrow \pm \infty} U(\xi)=A .
\end{gathered}
$$

Equation (20) implies that

$$
\begin{aligned}
& U \geq B_{1}, \quad \text { or } \quad U \leq B_{2}, \\
& \left(A-B_{1}\right)\left(A-B_{2}\right) \geq 0 .
\end{aligned}
$$

Since $3 A+2 c^{2} \neq 0$, introducing the constant $\alpha=(A /(3 A+$ $\left.\left.2 c^{2}\right)\right)$ yields

$$
\alpha(\alpha+1) \geq 0
$$

which implies that

$$
\alpha<-1, \quad \alpha=-1, \quad \alpha=0, \quad \alpha>0 .
$$

From the standard phase analysis, we know that if $U(\xi)$ is a single peak soliton solution of the $B(2,2)$ equation (3), then

$$
\begin{aligned}
& U^{\prime}=-\operatorname{sgn}(A) \frac{U-A}{2 U} \sqrt{\left(U-B_{1}\right)\left(U-B_{2}\right)} \operatorname{sgn}(\xi), \\
& U(0)=\left\{\begin{array}{cl}
\max \left(0, B_{1}\right), & \text { if } U(0) \text { is a minimum, } \\
\min \left(0, B_{2}\right), & \text { if } U(0) \text { is a maximum. }
\end{array}\right.
\end{aligned}
$$

Taking the integration of both sides of (24) leads to

$$
\int \phi(U) d U=-\frac{|\xi|}{2}
$$

where $\phi(U)=-\operatorname{sgn}(A)\left(U /(U-A) \sqrt{\left(U-B_{1}\right)\left(U-B_{2}\right)}\right)$. When $\alpha \neq 0,-1$, that is, $2 A\left(2 A+c^{2}\right) \neq 0$, we obtain

$$
\begin{aligned}
\int \phi(U) d U & =\operatorname{sgn}(A) I_{1}(U)+\frac{|A|}{\sqrt{4 A^{2}+2 A c^{2}}} I_{2}(U)-K \\
& =\Phi(U)-K,
\end{aligned}
$$

with

$$
\begin{aligned}
& I_{1}(U)=\ln \left|2 U-B_{1}-B_{2}+\sqrt{\left(U-B_{1}\right)\left(U-B_{2}\right)}\right| \\
& I_{2}(U)=\ln \left(\mid\left(\left(A-B_{1}\right)\left(U-B_{2}\right)+\left(A-B_{2}\right)\left(U-B_{1}\right)\right.\right. \\
& \left.\quad-2 \sqrt{\left(A-B_{1}\right)\left(A-B_{2}\right)\left(U-B_{1}\right)\left(U-B_{2}\right)}\right) \\
& \left.\quad \times(U-A)^{-1} \mid\right),
\end{aligned}
$$

and $K$ is an integration constant. Thus we obtain the implicit solution $U(\xi)$ defined by

$$
\Phi(U)=-\frac{|\xi|}{2}+K
$$


Obviously,

$$
\begin{aligned}
& I_{1}\left(B_{1}\right)=I_{1}\left(B_{2}\right)=\ln \left|B_{1}-B_{2}\right|=\ln \frac{\sqrt{6 A c^{2}+4 c^{4}}}{3}, \\
& I_{2}\left(B_{1}\right)=I_{2}\left(B_{2}\right)=\ln \left|B_{1}-B_{2}\right|=\ln \frac{\sqrt{6 A c^{2}+4 c^{4}}}{3} .
\end{aligned}
$$

So, for $U(0)=B_{1}$ or $B_{2}$, the constant $K_{0}=\Phi(U(0))$ is defined by

$$
\begin{aligned}
K_{0}= & \operatorname{sgn}(A) \ln \frac{\sqrt{6 A c^{2}+4 c^{4}}}{3}+\frac{|A|}{\sqrt{6 A c^{2}+4 c^{4}}} \\
& \times \ln \frac{\sqrt{6 A c^{2}+4 c^{4}}}{3},
\end{aligned}
$$

and for $U(0)=0$,

$$
K_{0}=I_{1}(0)-\frac{2 A}{\sqrt{4 A^{2}-2 A c}} I_{2}(0) .
$$

(1) $\alpha<-1$.

$$
\text { If } \alpha<-1 \text {, then }
$$

$$
A<B_{2}<0<B_{1}, \quad U(0)=B_{2}, \quad A<U \leq B_{2} .
$$

From $\phi(U)<0$, we know that $\Phi(U)$ is strictly decreasing on $\left(A, B_{2}\right]$, and

$$
\Phi_{1}(U)=\left.\Phi\right|_{\left(A, B_{2}\right]}(U)
$$

has the inverse denoted by $U_{1}(\xi)=\Phi_{1}^{-1}\left(-(|\xi| / 2)+K_{0}\right) . U_{1}(\xi)$ gives a smooth soliton solution satisfying

$$
U_{1}(0)=B_{2}, \quad \lim _{\xi \rightarrow \pm \infty} U_{1}(\xi)=A, \quad U_{1}^{\prime}(0)=0 .
$$

The profile of smooth soliton solution is shown in Figure 1(a).

(2) $\alpha=-1$.

If $\alpha=-1$, then $A=B_{2}=-c^{2} / 2$ and $B_{1}=c^{2} / 6$; there is no single peak soliton solution.

(3) $\alpha=0$.

In case $A=0$, (9) becomes

$$
U^{\prime}=-\frac{1}{2} \sqrt{U\left(U+\frac{4 c^{4}}{3}\right)} \operatorname{sgn}(\xi), \quad U( \pm \infty)=0 .
$$

Thus there is no single peak soliton solution for the previous boundary condition (8).

(4) $\alpha>0$.

If $\alpha>0$, then $A>0$ and

$$
B_{2}<B_{1}<0<A, \quad U(0)=0, \quad 0 \leq U<A .
$$

From $\phi(U)>0$, we know that $\Phi(U)$ is strictly increasing on $[0, A)$, and

$$
\Phi_{2}(U)=\left.\Phi\right|_{[0, A)}(U)
$$

gives a unique cusped soliton solution. Therefore, $U_{2}(\xi)=$ $\Phi_{2}^{-1}\left(-(|\xi| / 2)+K_{0}\right)$ is the solution satisfying

$$
\begin{aligned}
& U_{2}(0)=0, \quad \lim _{\xi \rightarrow \pm \infty} U_{2}(\xi)=A, \\
& U_{2}^{\prime}(0+)=+\infty, \quad U_{2}^{\prime}(0-)=-\infty .
\end{aligned}
$$

The profile of cuspon is shown in Figure 1(b).

Case II $\left(A=-2 c^{2} / 3\right)$. If $A=-2 c^{2} / 3$, then the only possible single peak soliton solution is the peakon

$$
U(\xi)=-\frac{2 c^{2}}{3}\left(1-e^{-|x-c t| / 2}\right) .
$$

The profile of peaked soliton is shown in Figure 1(c).

Case III $\left(A<-2 c^{2} / 3\right)$. In this case, according to Theorem 4 and standard phase portrait analytical technique, we have $U(0)=0, A<U \leq 0$, and

$$
U^{\prime}=\frac{U-A}{U} \sqrt{\frac{3 U^{2}+\left(6 A+4 c^{2}\right) U+3 A^{2}+2 A c^{2}}{12}} \operatorname{sgn}(\xi) .
$$

Let

$$
\begin{gathered}
X=U+a_{1}, \\
a_{1}=\frac{3 A+2 c^{2}}{3}, \\
a_{2}^{2}=-\frac{2}{9}\left(3 A+2 c^{2}\right),
\end{gathered}
$$

and then (41) becomes

$$
\varphi(X) d X=\frac{X-a_{1}}{\left(X-a_{1}-A\right) \sqrt{X^{2}+a_{2}^{2}}} d X=\frac{1}{2} \operatorname{sgn}(\xi) d \xi .
$$

Integration of both sides of (43) gives

$$
\Psi(X)=\frac{|\xi|}{2}+K
$$

where

$$
\begin{gathered}
\Psi(X)=J_{1}(X)+\frac{A}{\sqrt{\left(a_{1}+A\right)^{2}+a_{2}^{2}}} J_{2}(X), \\
J_{1}(X)=\ln \left|X+\sqrt{X^{2}+a_{2}^{2}}\right|,
\end{gathered}
$$

$J_{2}(X)$

$$
=\ln \left|\frac{2\left[a_{2}^{2}+\left(a_{1}+A\right) X-\sqrt{\left(a_{1}+A\right)^{2}+a_{2}^{2}} \sqrt{X^{2}+a_{2}^{2}}\right]}{X-a_{1}-A}\right| .
$$




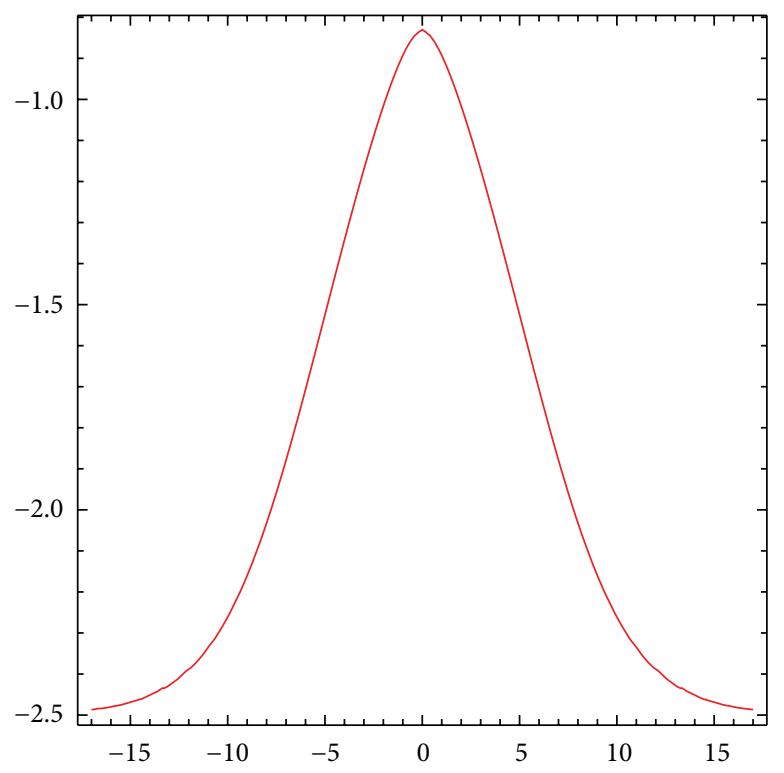

(a)

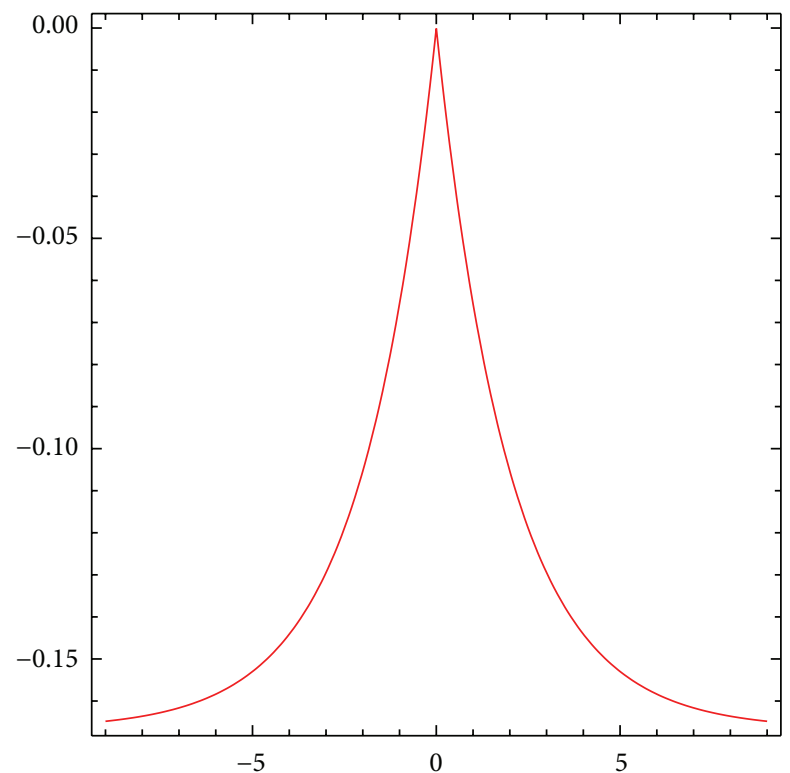

(c)

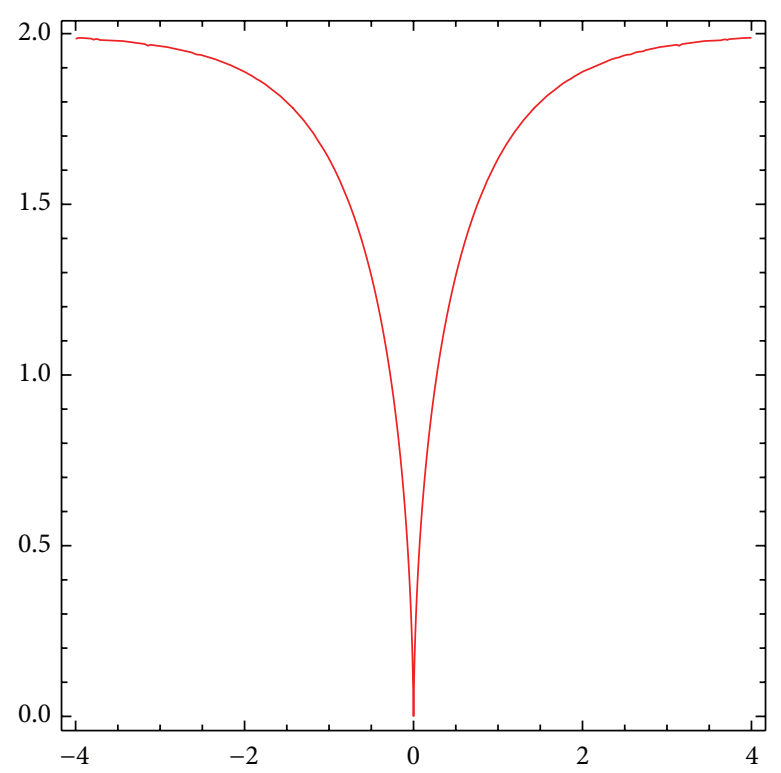

(b)

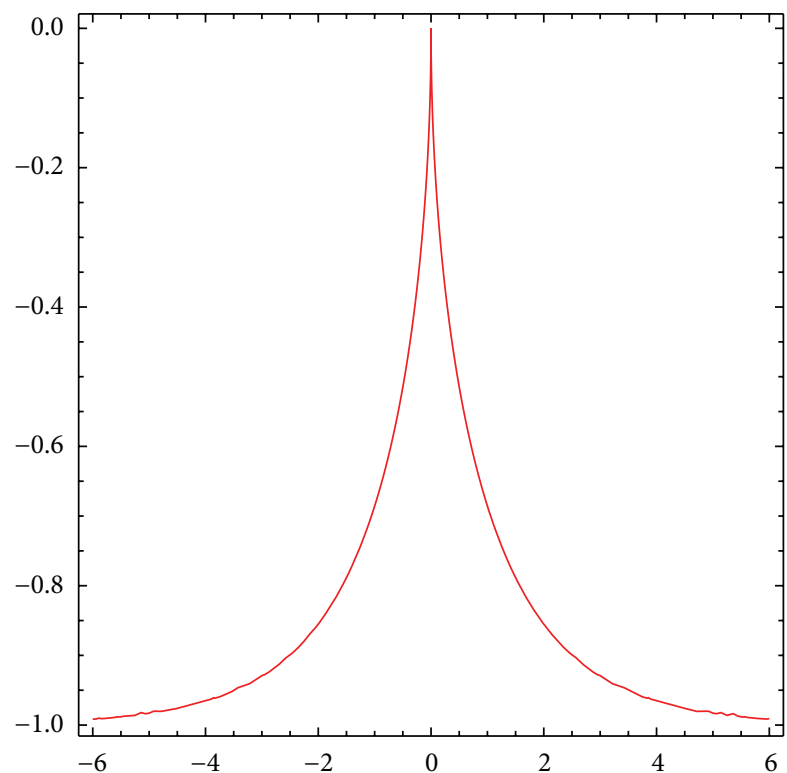

(d)

Figure 1: The profiles of waves. (a) Smooth soliton, $A=-5 / 2, c=-2$. (b) Cuspon, $A=2, c=1$. (c) Peakon, $A=-2 / 3, c=1$. (d) Cuspon, $A=-1, c=-1$.

$\Psi(X)$ is strictly decreasing on the interval $\left[a_{1}, a_{1}+A\right)$. Define

$$
\Psi_{1}(X)=\left.\Psi\right|_{\left[a_{1}, a_{1}+A\right)}(X)
$$

Then

$$
\Psi_{1}(X)=\frac{|\xi|}{2}+K_{0}
$$

where

$$
K_{0}=J_{1}\left(a_{1}\right)+\frac{A}{\sqrt{\left(a_{1}+A\right)^{2}+a_{2}^{2}}} J_{2}\left(a_{1}\right) .
$$

Since $\Psi_{1}$ is a strictly decreasing function, we can solve for $X$ uniquely from (47) and obtain

$$
U(\xi)=\Psi_{1}^{-1}\left(\frac{|\xi|}{2}+K_{0}\right)-a_{1},
$$

which satisfies

$$
\begin{gathered}
U(0)=0, \quad \lim _{\xi \rightarrow \pm \infty} U(\xi)=A, \\
U^{\prime}(0+)=-\infty, \quad U^{\prime}(0-)=+\infty .
\end{gathered}
$$


Therefore, the solution $U$ defined by (49) is a cusped soliton solution for the $B(2,2)$ equation (3). The profile of cuspon is shown in Figure 1(d).

Let us summarize our results in the following theorem.

Theorem 6. Suppose that $U(x-c t)$ is a single peak soliton solution for the $B(2,2)$ equation (3) at the peak point $\xi_{0}=$ 0 , which satisfies the inhomogeneous boundary condition (8). Then one has the following.

(1) For $3 A+2 c^{2} \neq 0$, let $\alpha=A /\left(3 A+2 c^{2}\right)$; then

(i) if $-1 \leq \alpha \leq 0$, there is no soliton for the $B(2,2)$ equation (3);

(ii) if $\alpha<0$ and $A<0$, the $B(2,2)$ equation (3) has the smooth soliton solution

$$
U(\xi)=\Phi_{1}^{-1}\left(-\frac{|\xi|}{2}+K_{0}\right)
$$

with the following properties:

$$
U(0)=B_{2}, \quad U( \pm \infty)=A, \quad U^{\prime}(0)=0 ;
$$

(iii) if $\alpha>0$, the $B(2,2)$ equation (3) has the cusped soliton solution

$$
U(x, t)=\Phi_{2}^{-1}\left(-\frac{|\xi|}{2}+K_{0}\right)
$$

with the following properties:

$$
\begin{gathered}
U(0)=0, \quad U( \pm \infty)=A, \\
U^{\prime}(0+)=+\infty, \quad U^{\prime}(0-)=-\infty ;
\end{gathered}
$$

(iv) if $\alpha<0$ and $A>0$, the $B(2,2)$ equation (3) has the cusped soliton solution

$$
U(x, t)=\Psi_{1}^{-1}\left(\frac{|\xi|}{2}+K_{0}\right)-a_{1},
$$

with the following properties:

$$
\begin{gathered}
U(0)=0, \quad U( \pm \infty)=A, \\
U^{\prime}(0+)=-\infty, \quad U^{\prime}(0-)=+\infty .
\end{gathered}
$$

(2) When $3 A+2 c^{2}=0$, then $U$ is the peakon

$$
U(x, t)=-\frac{2 c^{2}}{3}\left(1-e^{-|x-c t| / 2}\right)
$$

with the following properties:

$$
\begin{gathered}
U(0)=0, \quad U( \pm \infty)=A, \\
U^{\prime}(0+)=-\frac{c^{2}}{3}, \quad U^{\prime}(0-)=\frac{c^{2}}{3} .
\end{gathered}
$$

\section{Acknowledgments}

This work is supported by the National Natural Science Foundation of China (no. 11071222) and the Natural Science Foundation of Huzhou University (no. KX21061). The authors would like to thank the anonymous referees for their suggestions and comments which made the presentation of this work better. And the first author also wants to express her sincere gratitude to Professor Zhijun Qiao for his kind help.

\section{References}

[1] R. Camassa and D. D. Holm, "An integrable shallow water equation with peaked solitons," Physical Review Letters, vol. 71, no. 11, pp. 1661-1664, 1993.

[2] A. S. Fokas, "On a class of physically important integrable equations," Physica D, vol. 87, pp. 145-150, 1995.

[3] B. Fuchssteiner, "Some tricks from the symmetry-toolbox for nonlinear equations: generalizations of the Camassa-Holm equation," Physica D, vol. 95, no. 3-4, pp. 229-243, 1996.

[4] P. J. Olver and P. Rosenau, "Well-posedness and blow-up solutions for an integrable non-linearly dispersive model wave equation," Physical Review E, vol. 53, pp. 1900-1906, 1996.

[5] Z. Qiao, "A new integrable equation with cuspons and W/Mshape-peaks solitons," Journal of Mathematical Physics, vol. 47, no. 11, Article ID 112701, 9 pages, 2006.

[6] Z. Qiao and X. Li, "An integrable equation with nonsmooth solitons," Theoretical and Mathematical Physics, vol. 167, no. 2, pp. 584-589, 2011.

[7] Z. Qiao, B. Xia, and J. Li, "Integrable system with peakon, weak kink, and kink-peakon interactional solutions," 2012, http:// arxiv-web3.library.cornell.edu/pdf/1205.2028v2.pdf.

[8] V. Novikov, "Generalizations of the Camassa-Holm equation," Journal of Physics A, vol. 42, no. 34, Article ID 342002, 2009.

[9] A. N. Hone and J. Wang, "Integrable peakon equations with cubic nonlinearity," Journal of Physics A, vol. 41, no. 37, Article ID 372002, 2008.

[10] Z.-Y. Yan, "New similarity reductions and compacton solutions for Boussinesq-like equations with fully nonlinear dispersion," Communications in Theoretical Physics, vol. 36, no. 4, pp. 385390, 2001.

[11] Z. Yan, "New families of solitons with compact support for Boussinesq-like $B(m, n)$ equations with fully nonlinear dispersion," Chaos, Solitons \& Fractals, vol. 14, no. 8, pp. 1151-1158, 2002.

[12] Z. Yan and G. Bluman, "New compacton soliton solutions and solitary patterns solutions of nonlinearly dispersive Boussinesq equations," Computer Physics Communications, vol. 149, no. 1, pp. 11-18, 2002.

[13] Y. Zhu and C. Lu, "New solitary solutions with compact support for Boussinesq-like $B(2 n, 2 n)$ equations with fully nonlinear dispersion," Chaos, Solitons \& Fractals, vol. 32, no. 2, pp. 768772, 2007.

[14] Y. Shang, "New exact special solutions with solitary patterns for Boussinesq-like $B(m, n)$ equations with fully nonlinear dispersion," Applied Mathematics and Computation, vol. 173, no. 2, pp. 1137-1148, 2006.

[15] L. Zhang, L.-Q. Chen, and X. Huo, "New exact compacton, peakon and solitary solutions of the generalized Boussinesqlike $B(m, n)$ equations with nonlinear dispersion," Nonlinear Analysis A, vol. 67, no. 12, pp. 3276-3282, 2007. 
[16] Z. J. Qiao and G. P. Zhang, "On peaked and smooth solitons for the Camassa-Holm equation," Europhysics Letters, vol. 73, no. 5, pp. 657-663, 2006.

[17] G. Zhang and Z. Qiao, "Cuspons and smooth solitons of the Degasperis-Procesi equation under inhomogeneous boundary condition," Mathematical Physics, Analysis and Geometry, vol. 10, no. 3, pp. 205-225, 2007.

[18] A. Chen and J. Li, "Single peak solitary wave solutions for the osmosis $K(2,2)$ equation under inhomogeneous boundary condition," Journal of Mathematical Analysis and Applications, vol. 369, no. 2, pp. 758-766, 2010. 


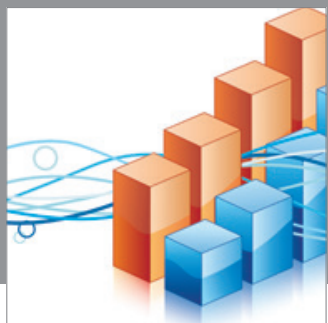

Advances in

Operations Research

mansans

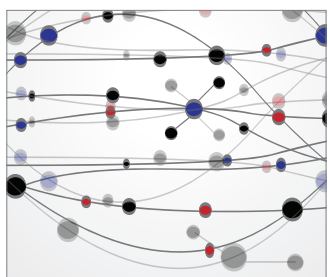

The Scientific World Journal
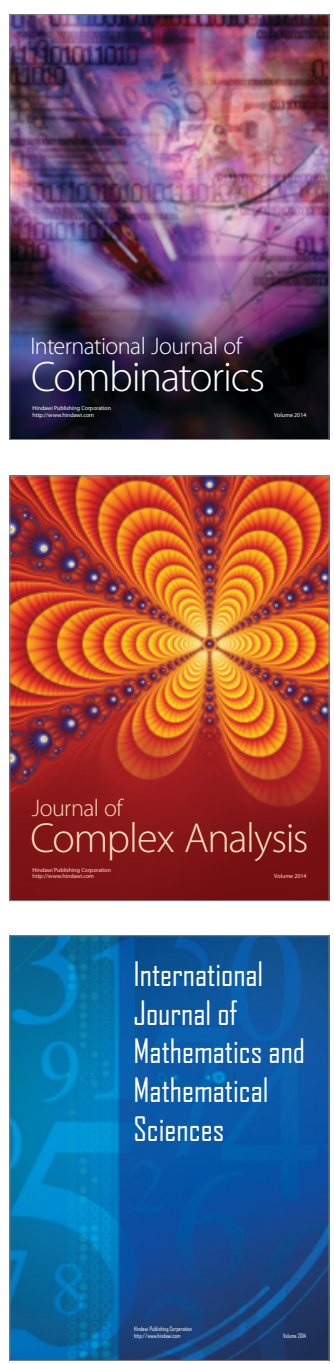
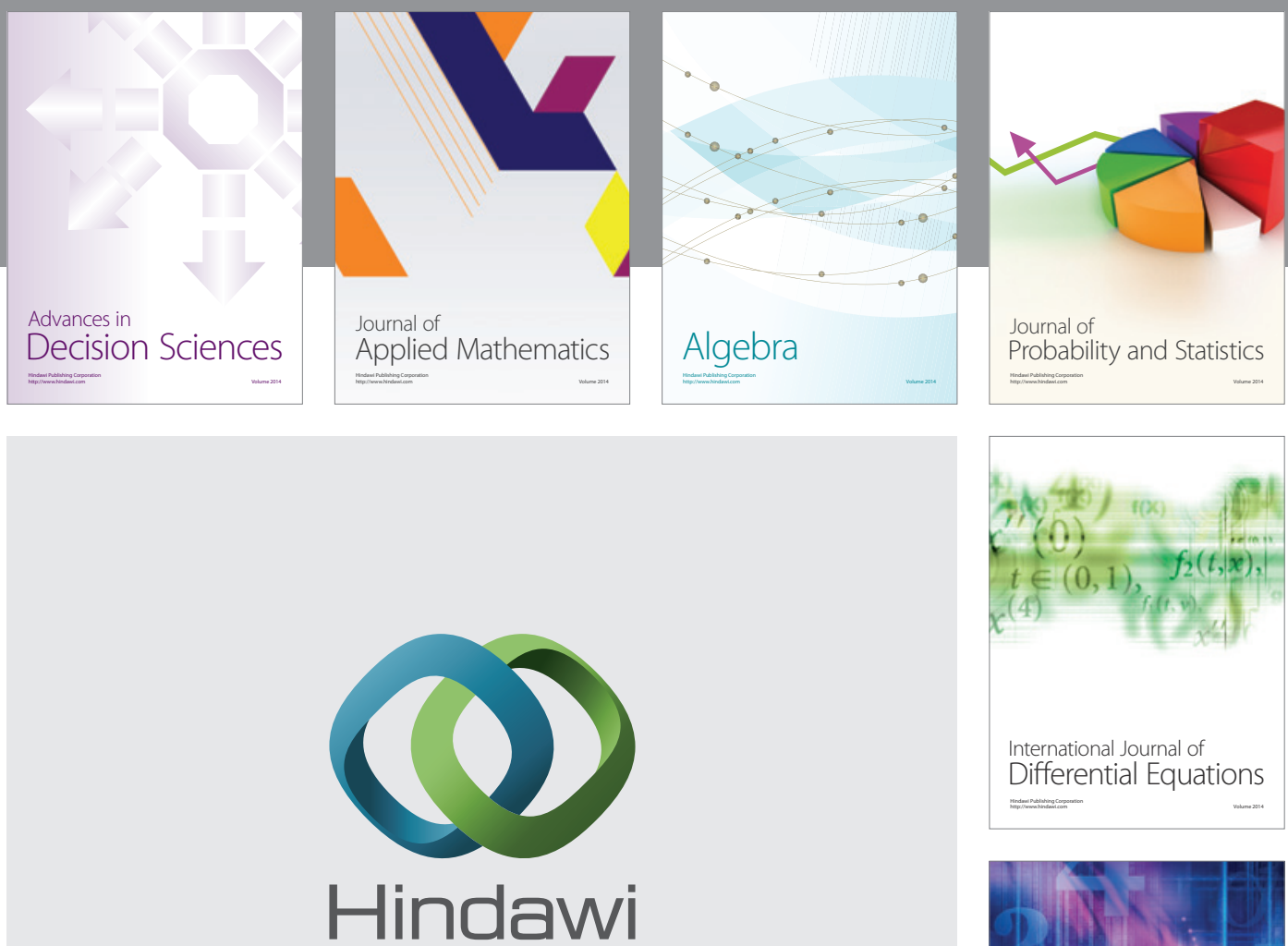

Submit your manuscripts at http://www.hindawi.com
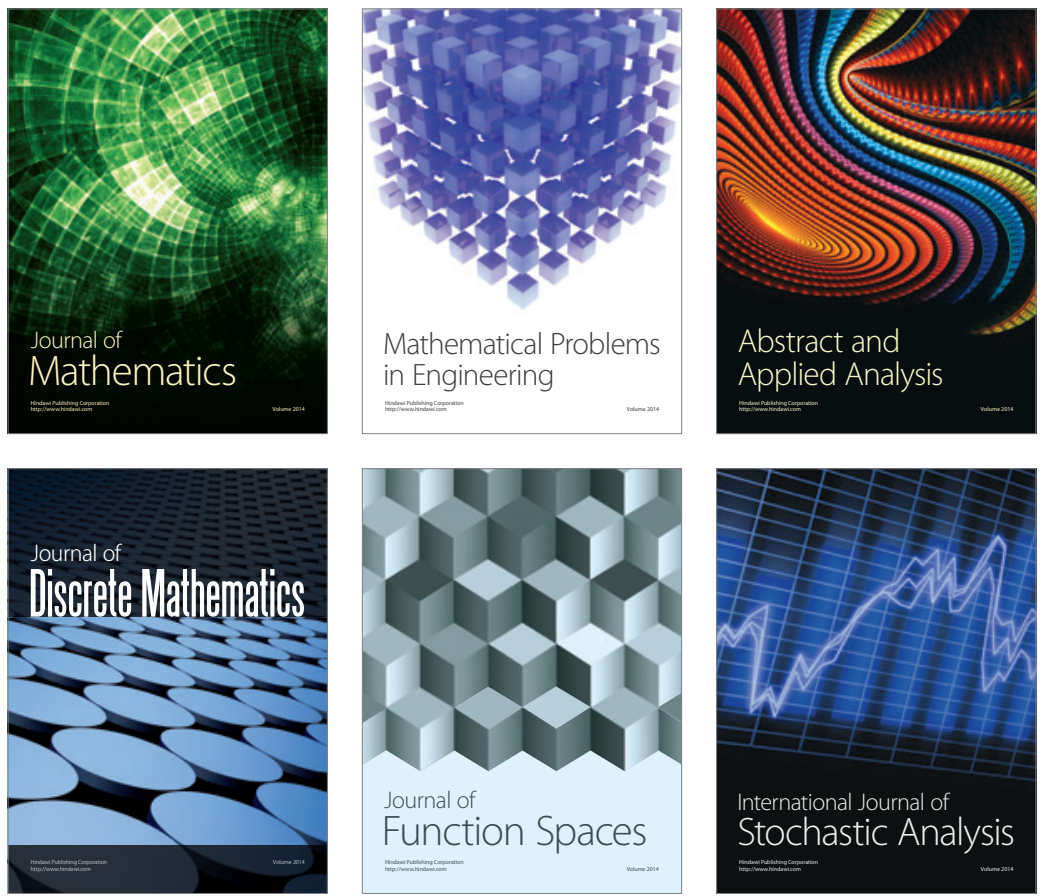

Journal of

Function Spaces

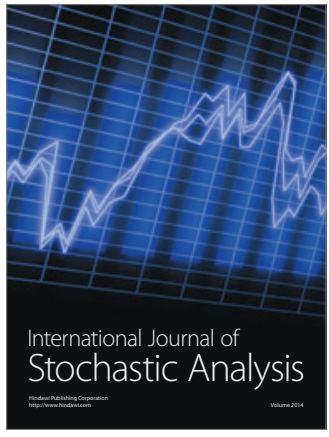

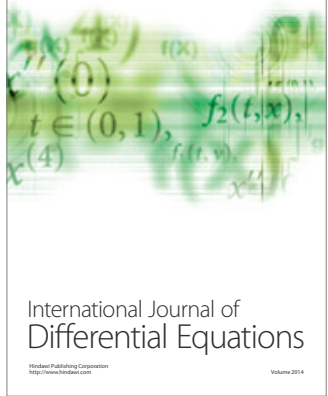
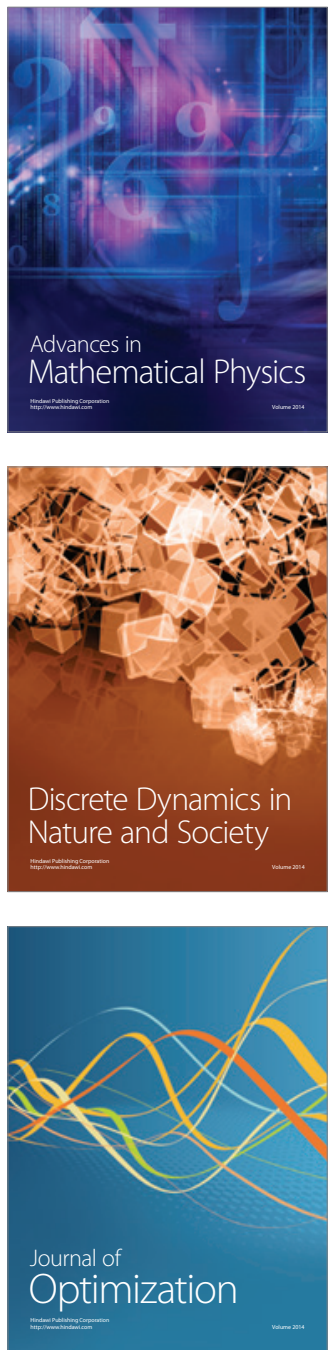